\title{
HELMINTHES PARASITES DES CHIENS ET DES CHATS EN EGYPTE
}

Par Abdel AZIM (1)

En étudiant le problème de l'incidence de l'Echinococcus granulosus et ses relations avec la maladie hydatique de l'homme, j'ai eu l'occasion de disséquer beaucoup d'animaux venant de localités différentes du pays. Les chiens ramassés représentaient tous les âges et tous les pedigrees de la population canine qui fréquente les rues et se nourrit des résidus des maisons et des marchés. Les chats ont été capturés seulement dans les rues du Caire.

Les animaux apportés au laboratoire étaient immédiatement sacrifiés et l'abdomen était ouvert: l'intestin était séparé du pylore au caecum et fendu sur toute sa longueur. La muqueuse était grattée et le produit de ce grattage transporté dans de hauts cylindres de verre où il était vigoureusement agité avec de l'eau pour briser les débris et libérer des villosités les petits parasites. Tout le matériel était alors passé sur un tamis à 10 mailles par pouce linéaire $(2 \mathrm{~cm} ., 54)$. Les plus gros vers étaient retenus par ce tamis et recueillis à sa surface. Le filtrat, renfermant les parasites plus petits, était mis à sédimenter, puis le liquide surnageant était rejeté. Le dépôt était enfin lavé à plusieurs reprises et, par ce moyen, on récoltait tous les petits parasites qui s'y trouvaient. Les autres organes étaient alors examinés et on faisait des gouttes épaisses avec le sang des cavités cardiaques.

Les parasites intestinaux des chiens ont fait l'objet d'une discussion dans un précédent article. Des statistiques des parasites des chiens et des chats ont été établies dans différents pays et ont montré qu'environ 170 espèces de parasites ont été rencontrées chez ces animaux.

Le tableau I donne le nombre d'espèces de vers parasites mentionné dans diverses statistiques.

Sur 320 chiens examinés, 99 p. 100 , et sur 150 chats, 100 p. 100 , hébergeaient un ou plusieurs parasites. En moyenne, un animal

(1) Traduit de l'anglais par le $\mathrm{D}^{r}$ Maurice Langeron.

AnNales de Parasitologie, T. XVII, No 1 - $-1^{\text {er }}$ janvier 1939, p. 32-36. 
TABLEAU I

\begin{tabular}{|c|c|c|c|c|}
\hline PAYS & Autevi & ANNÉE & Chiens & Cнатs \\
\hline Shanghai (Chine).... & Andrews & 1937 & 17 & 13 \\
\hline Calcutta (Inde)...... & Chandler & 1925 & - & 12 \\
\hline Manaos (Brésil)..... & Gordon et Young & 1922 & 7 & 3 \\
\hline 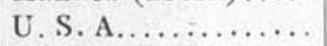 & Hall & 1923 & 28 & 13 \\
\hline Sibérie orientale.... & Issaitschikoff & 1925 & 9 & 9 \\
\hline Aberystwyth (Wales) & Lewis & 1927 & 7 & 9 \\
\hline Cambridge .......... & Nuttall et Strickland & 1908 & 4 & - \\
\hline Varsovie............ & Penkacki & 1929 & 18 & - \\
\hline Sakhalin......... & Petroff & 1928 & 10 & 7 \\
\hline Arménie ........... & Popoff & 1924 & 11 & 9 \\
\hline Russie du Sud....... & Skrjabin & 1924 & 19 & 23 \\
\hline Marseille........... & Solomon & 1933 & 8 & - \\
\hline Iles Philippines..... & Wharton & 1917 & 6 & - \\
\hline Palestine............ & Witenberg & 1934 & 35 & 28 \\
\hline Egypte............ & Abdel Azim & 1938 & 29 & 27 \\
\hline
\end{tabular}

était porteur de 4 parasites. La plupart de ces parasites se trouvaient dans le tube digestif ; il n'y en avait aucun dans le foie ou dans la cavité générale. Dirofilaria n'a été vu qu'une fois et Füllebornius medinensis deux fois.

Les parasites trouvés en Egypte chez les chiens et les chats sont énumérés dans le tableau II.

Le parasite le plus commun chez les chiens examinés était Toxascaris leonina, qui a été trouvé dans 73 p. 100 des chiens. Chez le chat, le plus commun était Heterophyes heterophyes, qui a été trouvé dans 65 p. 100 des cas.

Trématodes. - Onze espèces d'hétérophyidés sont libéralement représentées chez les chiens et les chats. Bien qu'elles appartiennent à 5 genres différents, leur biologie est la même : les poissons sont leur second hôte intermédiaire. 75 p. 100 des chiens d'Alexandrie et 59 p. 100 de ceux du Caire hébergent une ou plusieurs de ces espèces. Ceux de la Haute-Egypte, où on ne consomme que du poisson d'eau douce, ne renferment que des Haplorchis. Le fait que 90 p. 100 des chats sont infestés par différentes espèces d'hétérophyidés est certainement dû à la préférence de ces animaux pour le poisson. Un chat ne refuse jamais un repas de poisson, tandis 
TABLEAU II

\begin{tabular}{|c|c|c|}
\hline & Chirns & Cents \\
\hline \multicolumn{3}{|l|}{ Trématodes } \\
\hline 1. Diplostomum teregenna Gohar $1932 \ldots \ldots \ldots \ldots$ & + & + \\
\hline 2. Diplostomum azimi Gohar $1932 \ldots \ldots \ldots \ldots \ldots$ & - & + \\
\hline 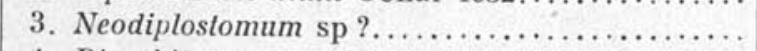 & + & - \\
\hline 4. Diorchilrema pseudocirralum Witenberg 1929 . & + & + \\
\hline 5. Echinochasmus lilipulanus (Looss 1896)....... & + & + \\
\hline 6. Echinoparyphinm recurvatum Linstow $1873 \ldots$ & $+($ ex. $)(1)$ & - \\
\hline 7. Haplorchis pumilio (Looss 1896)............. & + & + \\
\hline 8. Haplorchis H. laichui (Nishigori 1924)....... & + & + \\
\hline 9. Haplorchis H. yokogawai Natsuta $1932 \ldots \ldots$. . & + & + \\
\hline 10. Ascocotyle coleoslomum Looss $1899 . . . . . . .$. & + & - \\
\hline 11. Heterophyes heterophyes Siebold $1852 \ldots \ldots \ldots$ & + & + \\
\hline 12. Heterophyes $H$. aqualis Looss $1902 \ldots \ldots \ldots \ldots$. . . & + & + \\
\hline 13. Heterophyes $H$. dispar Looss $1902 \ldots \ldots \ldots \ldots$ & + & + \\
\hline 14. Parascocotyle ascalonga Witenberg $1929 . \ldots$. & + & + \\
\hline 15. Parascocolyle P. italica Alessandrini $1906 \ldots \ldots$ & + & - \\
\hline 16. Parascocolyle P. longa (Ransom 1920)........ & + & + \\
\hline 17. Prohemislomum vivax (Sonsino 1893 )......... & + & + \\
\hline 18. Pygidiopsis genala Looss $1 \subseteq 07 \ldots \ldots \ldots \ldots \ldots \ldots$ & + & + \\
\hline 19. Sliclodora sauvakinensis Looss $1899 \ldots \ldots \ldots \ldots$ & + & $T$ \\
\hline \multicolumn{3}{|l|}{ Cestodes } \\
\hline 20. Diplopylidium acantholetra Parona $1886 \ldots . .$. & - & + \\
\hline 21. Diplopylidium nölleri (Skriabin 1924)......... & - & + \\
\hline 22. Dipylidium caninum Linné $1753 \ldots \ldots \ldots \ldots \ldots$ & + & + \\
\hline 23. Echinococcus granulosus Batch $1786 . \ldots \ldots \ldots$. & + & $+($ ex. $)(1)$ \\
\hline 24. Joyeuxia pasquali (Diamare 1893)............. & - & + \\
\hline 25. Reditania tanixformis (Batch 1786).......... & - & + \\
\hline 26. Tzenia hydatigena Pallas $1766 \ldots \ldots \ldots \ldots \ldots$ & + & + \\
\hline \multicolumn{3}{|l|}{ Nématodes } \\
\hline 27. Ancylostoma caninum Ercolani $1859 \ldots \ldots \ldots \ldots$ & + & + \\
\hline 28. Chlamydonema præputiale (Linstow 1884).... & - & + \\
\hline 29. Dirofilaria immitis (Leidy 1856$) \ldots \ldots \ldots \ldots \ldots$ & + & - \\
\hline 30. Füllebornius medinensis (L. 1758)............ & + & - \\
\hline 31. Rictularia cahirensis Jägerskiöld $1924 \ldots \ldots \ldots$ &.+ & + \\
\hline 32. Spirocerca sanguinolenla (Rud. 1819).......... & $+($ ex. $)(1)$ & - \\
\hline 33. Toxascaris leonina (Linstow 1902).......... & + & + \\
\hline 34. Uncinaria stenocephala (Raillet 1884)......... & & - \\
\hline Acanthocéphales & & \\
\hline $\begin{array}{l}\text { 35. Echinorhynchus pachyacanthus (Sonsino 1889). } \\
\text { 36. Centrorhynchus clitorideus (Meyer 1931)....... }\end{array}$ & + & - \\
\hline
\end{tabular}

(1) (Ex.) indique que l'infection a été obtenue seulement expérimentalement, 
que certains chiens ne consentent à le manger que s'ils sont affamès ou si le poisson est déjà cuit.

Gestodes. - Dipylidium caninum existe chez 65 p. 100 des chiens et 50 p. 100 des chats. Dans les deux cas, on le trouve toujours en grand nombre. Les autres dipylidinés n'existent que chez les chats. Taenia hydatigena a été trouvé chez 23 p. 100 des chiens et rarement chez les chats. Reditaenia existait chez 10 p. 100 des chats et la larve (Cysticercus fasciolaris) a été vue quelquefois dans le foie de rats blancs.

Echinococcus granulosus est très important au point de vue de la maladie hydatique de l'homme. Il n'est pas uniformément répandu, car son incidence dépend beaucoup du contrôle et de l'inspection des viandes. Il a été trouvé dans 3 p. 100 des chiens du Caire, dans 2 p. 100 de ceux d'Alexandrie et dans 10 p. 100 de ceux de Haute-Egypte, où les bestiaux et les chameaux infectés sont abattus dans des marchés de village; dans ces endroits, on laisse les chiens dévorer les issues et les débris de carcasses. L'infection par Echinococcus granulosus a été obtenue expérimentalement chez le chat.

Nématodes. - Toxocara n'a été rencontré dans aucun cas. Rictularia cahirensis a été trouvé dans 8 p. 100 des chats et jamais chez les chiens. Spirocerca n'a jamais été vu dans la nature, mais l'infection a été obtenue expérimentalement en faisant avaler à des chiens des larves enkystées dans le péritoine de hérissons et de lézards. Füllebornius medinensis a été trouvé deux fois dans le tissu conjonctif des pattes de chiens.

\section{BiBLIOGRAPHIE}

Abdel Azim (M.). - On the identity and development of Echirostomum recurvatum Linstow 1873. Ann. Trop. Med. and Parasit., XXIV, $\mathrm{n}^{\circ} 2,1930$.

- On Prohemistomum vivax (Sonsino 1892) and its development from Cercaria vivax (Sonsino 1892). Zeitschr. f. Paras., V, 1933, p. 432-436.

- On the intestinal helminths of dogs in Egypt. Jour. Egypt. Med. Assoc., XXI, $\mathrm{n}^{\circ} 3,1938$.

Andrews (N. M.). - The helminths parasites of dogs and eats in Shanghai. China. Jour. Helminths, V, $\mathrm{n}^{\circ} 2,1937$.

Chander (A.). - The helminths of cats in Calcutta and the relation of cats to human helminthic infection. Ind. Jl. Med. Res., III, 1925, p. 213-228.

Gordon (R. M.) et Young (C. J.). - Parasites of dogs and cats in Amazonas. Ann. Trop. Med. and Parasit., XIV, 1922, p. 287-300. 
HaLl (M. C.). - Internal parasites of dogs and cats in the United States. $J l . A m$. Vet. Assoc., LXIII, 1923, p. 11-51.

LEwis (E. A.). - The study of the helminths of dogs and cats Aberystwyth, Wales. Jl. Helminth., V, 1927, p. 171-182.

Nuttall (G.) et Strickland. - Note on the prevalence of intestinal worms in Cambridge. Parasitology, I, $\mathrm{n}^{\circ} 3,1902$.

PrNkacki (C.). - Recherches sur les vers parasités dans l'intestin du chien. Bull. Acad. Pol. Sc. et Lettres, Cracovie, 1929, p. 215-227.

PEtroff (A. M.). - A contribution to the knowledge of the fauna of parasitic worms of domestic earnivores in Russian Turkestan (en russe). QEuvres Irst. Med. Vet. Exp., Moscou, II, 1926, p. 86-101.

PopofF (N. P.). - Parasitic worms of domestic carnivores in Armenia (en russe). Trudi Trop. Inst. Armenii, I, 1924, 8 p.

Sкrлaвin (K. I.). - Zur Charakteristik der Wurminvasionen bei Hunden und Katzen des Dongebietes. Berl. Tierarzll. Wochenschr., XL, 1924, 8 p.

Solomon (G.). - The helminth parasites of dogs in Marseille. Jl. Helm., II, 1933 , p. $157-162$.

Wharton (L.). - The intestinal worms of dogs in the Phillipine Islands. Jl. Parasit., IV, $\mathrm{n}^{\circ} 2,1917$.

Witenberg (G.). - Parasitic worms of dogs and eats in Palestine. Veter. Record, XIV, $\mathrm{n}^{\circ} 9,1934$.

Reptilien als Zwischenwirte Parasitischer Würmer von Katze und Hunde. Tierarztl. Rundschau, XXXIV, 1928, p. 607.

Ueber zwei in Palestina in Hunden und Katzen Parasitierende Echinochasmusarten (Trematoda). Zeitschr. f. Parasitenkurde, V, 1932, p. 213216.

On the cestode subfamily Dipylidiinæ Stiles. Zeilschrift $f$. Parasitenkunde, IV, 1932 , p. 542-584.

Zur Kenntnis der Verbreitung von Echinokoklius und Trichinen in Palastina. Arch. f. Schiffs Troppenhyg., XXXVII, 1933, p. 37-41.

Research Instilute, Ministry of Health, Egypt. (Director: Prof. Dr. M. Khalil Bey). 